FACULDADE DE CIÊNCIAS ECONÔMICAS DA UFRGS Análise
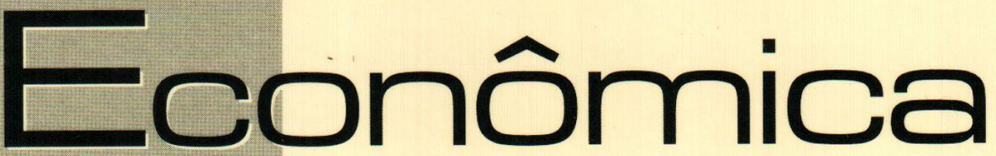

FLUTUACÃO CAMBIAL E METAS INFLACIONÁRIAS EM

ECONOMIAS EMERGENTES

OTAVIANO CANUTO E MárCIO HOLLAND

ATEORIA DE FORMACĆOO DE PRECOS E A TEORIA DOS PREÇOS DE

PRODUCAO

LOIZALBERTO ESTEVES

AECONOMIA EVOLUCIONISTA: UM CAPITULOSISTEMMICO DA

TEORIA ECONOMICA?

HUGOE. A. DA GAMACERQUEIRA

RELAÇ̃O INFLAÇÃO-PRODUTO NO BRASIL NO PERIODO PÓS-

REAL

MARCOSC. HOLANDA

DIVIIDA PÚBLICAMOBILLÍRIA FEDERAL BRASILEIRA: HISTÓRIA RECENTE E PERSPECTIVAS EXPLOSIVAS

ROGÉRIO MEURER E ROBERT WAYNE SAMOHYI

A CEPAL EA INTEGRACÃO REGIONAL LATINO-AMERICANA JACQUELINE A. HERNANDEZ HAFFNER

POPPER, HAYEK EA (IM)POSSIBILIDADE DE PREDIÇŌES ESPECIFICAS EM CIEENCIAS SOCIAIS

BRENA PAULA MAGNO FERNANDEZ

DINÂMICA RECENTE DO PROCESSO DE INCUBACG̃O DE

EMPRESAS DE BASE TECNOLOGICA NO BRASIL

EDUARDO GONÇALVES

TRIBUTACĀO COM SACRIF́CIO EQUUITATIVO: O CASO DO IMPOSTO DE RENDA PESSOA FISICA

STEFANO FLORISSI E EDUARDO PONTUAL RIBEIRO

A NOVATEORIA DO IMPÉRIOE AS VELHAS TEORIAS DO

IMPERIALISMO

GENTIL CORAZZA

Ô ABREALAS - A NOVA INSERÇÃO DO BRASIL NA ECONOMIA MUNDIAL

EMLLLANO LUIS KLEN

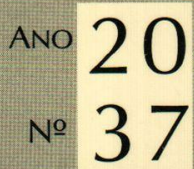

Março, 2002 
UNIVERSIDADE FEDERAL DO RIO GRANDE DO SUL

Reitora: Profa. Wrana Maria Panizzi

FACULDADE DE CIÊNCIAS ECONÔMICAS

Diretor: Prof. Pedro Cézar Dutra Fonseca

CENTRO DE. ESTUDOS E PESQUISAS ECONÔMICAS

Diretor: Prof. Gentil Corazza

DEPARTAMENTO DE CIÊNCIAS ECONÔMICAS

Chefe: Prof. Luiz Alberto Oliveira Ribeiro de Miranda

DAPARTAMENTO DE CIÊNCIAS CONTÁBEIS E ATUARIAIS

Chefe: João Marcos Leão da Rocha

CURSO DE PÓS-GRADUAÇÃO EM ECONOMIA

Coordenador: Prof. Eduardo Pontual Ribeiro

CURSO DE PÓS-GRADUAÇÃO EM ECONOMIA RURAL

Coordenador: Prof Jalcione Almeida

CONSELHO EDITORIAL: Carlos G. A. Mielitz Netto (UFRGS), Eduardo A. Maldonado Filho (UFRGS), Eduardo P. Ribeiro (UFRGS), Eleutério F. S. Prado (USP), Eugênio Lagemann (UFRGS), Fernando Cardim de Carvalho (UFRJ), Fernando Ferrari Filho (UFRGS), Fernando de Holanda Barbosa (FGV/RJ), Flávio Vasconcellos Comim (UFRGS), Gentil Corazza (UFRGS), Giácomo Balbinotto Neto (UFRGS), Gustavo Franco (PUC/RJ), Jan A. Kregel (Università di Bologna e John Hopkins University), João Rogério Sanson (UFSC), Joaquim Pinto de Andrade (UnB), Jorge Paulo Araújo (UFRGS), Juan H. Moldau (USP), Marcelo S. Portugal (UFRGS), Maria Alice L.ahorgue (UFRGS), Paul Davidson (University of Tennessee), Paulo Dabdab Waquil (UFRGS), Pedro Cézar Dutra Fonseca (UFRGS), Philip Arestis (South Bank University), Roberto C. Moraes (UFRGS), Ronald Otto Hillbrecht (UFRGS), Sabino da Silva Porto Jr. (UFRGS), Stefano Florissi (UFRGS), Werner Baer (Univ, of Illinois at Urbana-Champaign).

COMISSÃO EDITORIAL: Eduardo Augusto Maldonado Filho, Fernando Ferrari Filho, Gentil Corazza, Marcelo Savino Portugal, Paulo Dabdab Waquil; Roberto Camps Moraes.

EDITOR: Fernando Ferrari Filho

EDITOR ADJUNTO: Gentil Corrazza

SECRETÁRIA: Vanessa Hoffmann de Quadros

REVISÃO DE TEXTOS: Vanete Ricacheski

FUNDADOR: Prof. Antônio Carlos Santos Rosa

Os materiais publicados na revista Análise Econômica são da exclusiva responsabilidade dos autores. É permitida a reprodução total ou parcial dos trabalhos, desde que seja citada a fonte. Aceita-se permuta com revistas congêneres. Aceitam-se, também, livros para divulgação, elaboração de resenhas e recensões. Toda correspondência, material para publicação (vide normas na terceira capa), assinaturas e permutas devem ser dirigidos ao seguinte destinatário:

PROF FERNANDO FERRARI FILHO

Revista Análise Econômica - Av. João Pessoa, 52 CEP 90040-000 PORTO ALEGRE - RS, BRASIL Telefones: (051) 316-3348 e 316-3440 - Fax: (051) 316-3990

E-mail: rae@vortex ufrgs br

Análise Econômica

Ano 20, n 37 , março, 2002 - Porto Alegre

Faculdade de Ciências Econômicas, UFRGS, 2000

Periodicidade semestral, março e setembro.

1. Teoria Econômica - Desenvolvimento Regional Economia Agrícola - Pesquisa Teórica e Aplicada -

Periódicos. I. Brasil.

Faculdade de Ciências Econômicas,

Universidade Federal do Rio Grande do Sul.

CDD 330.05

CDU 33 (81) (05) 


\title{
A teoria de formação de preços e a teoria dos preços de produçãol
}

Luiz Alberto Esteves*

Resumo: Este trabalho procura analisar duas perspectivas muito conhecidas na teoria econômica: a primeira corresponde à teoria dos preços de produção desenvolvida por Sraffa, junto com a noção de longo período desenvolvida pela escola Neo-Ricardiana; a segunda corresponde às teorias de formação de preços em oligopólio (preços de mark-up), de crescimento econômico e da distribuição da renda desenvolvidas pela escola Pós-Keynesiana. Os objetivos deste trabalho consistem na análise dos esforços de conciliação destas abordagens distintas (com o objetivo de fornecer uma teoria geral para o princípio da demanda efetiva), sublinhando algumas críticas, assim como certas incompatibilidades deste procedimento.

Palavras-chave: crescimento econômico; distribuição de renda; demanda efetiva.

\begin{abstract}
This paper aims to analyze two well-know views of economic theory: the first one refers to the theory of the prices of production as developed by Sraffa combined with the notion of long-run developed by the Neo-Ricardian school; the second one refers to the price formation in oligopoly (mark-up prices), as well as to the theory of economic growth and income distribution developed by the Post-Keynesian school. We analyze in this paper some efforts made to conciliate these two approaches (in order to developed a general theory for the principle of effective demand), highlighting some criticism and some intellectual incompatibilities in this pursuit.
\end{abstract}

Key words: economic growth; income distribution; effective demand.

\footnotetext{
- Universidade Federal do Paraná, Setor de Ciências Sociais Aplicadas, Departamento de Economia.

${ }^{1} \mathrm{O}$ autor é grato aos comentários e sugestões do parecerista anônimo.
} 
O objeto de estudo deste trabalho consiste na análise de duas proposições muito conhecidas na teoria econômica: a primeira corresponde à teoria dos preços de produção desenvolvida por Sraffa; e a segunda corresponde à teoria de formação de preços em oligopólio (preços de markup) desenvolvida pelos pós-keynesianos da tradição Kalecki/Steindl.

$\mathrm{Na}$ abordagem de Kalecki existe uma separação entre as decisões de formação de preços de investimento, no sentido em que os preços são fixados sem qualquer relação direta com o financiamento do investimento. Uma segunda característica é a orientação de curto prazo das decisōes de formação de preços.

A abordagem Sraffiana estabelece um exemplo de teoria de preços (como oposição à teoria de formação de preços). Esta abordagem está primeiramente focalizada nos preços de equilíbrio de longo prazo, ou seja, em um vetor de preços que equalize a taxa de lucro nos vários setores da economia ou que corresponda às condiçōes ideais de reprodutibilidade de um sistema econômico capitalista.

Pode-se verificar que a metodologia utilizada por Kalecki diverge completamente da prescrita por Sraffa. Kalecki atribuía ao capitalismo a propriedade de ser um sistema "dinâmico", não demonstrando em qualquer momento a preocupação em formular uma teoria compatível com uma situação de estado estacionário (que segundo Kalecki, não envolve a noção de dinâmica) ou que correspondesse às condições ideais de reprodutibilidade de um sistema econômico capitalista, como faz Sraffa.

Os objetivos deste trabalho consistem em: (a) analisar o modelo multissetorial neo-Kaleckiano desenvolvido por Dutt (1987), que estabelece uma situação de longo prazo no qual é observada uma equalização da taxa de lucro entre os setores, através da formação de preços por markup; (b) analisar as críticas neo-Ricardianas a este modelo; e (c) aportar algumas incompatibilidades quando se procura sintetizar a teoria da formação de preços pós-keynesiana com a teoria dos preços de produção de Sraffa. 


\section{A teoria pós-keynesiana de formação de preços e a teoria clássica dos preços de produção}

Nesta seção procurar-se-á estabelecer de forma resumida o esclarecimento para as noções, diferenças e principais características da teoria de formação de preços e a teoria dos preços de produção, ou simplesmente, teoria dos preços.

Verificar-se-á ainda que a teoria da formação de preços estará associada à noção de curto prazo, enquanto a teoria dos preços de produção estará associada à noção do longo período. Segundo Sawyer (1994), a teoria pós-keynesiana estabelece uma "teoria de formação de preços", que pode ser definida conforme as seguintes características:

A notable feature of Kalecki's approach (as compared with many other postKeynesian approaches) is the separation between pricing and investment decisions, in the sense that prices are set without any direct regard the financing of investment. Investment decisions in the sense that the profit and output (and changes in output) which arise as a consequence of pricing are importante on investment. The second (and related) feature is the short-term orientation of pricing decisions. (SAWYER, 1994.a, p.214).

Quanto à abordagem clássica, esta pode ser considerada como uma "teoria dos preços", que é estabelecida com as seguintes características:

The Sraffian approach provides the example of a theory of price (as opposed to pricing). Four our purposes the key festures of this approach are firstly the focus on long-period equilibrium prices, which may be viewed as centres of gravity around which actual market prices fluctuate. There is no concern with the way by which prices are set in the short-term nor with the process by which the long-period equilibrium is reached. Secondly, there is mobility of capiatl generating a tendency towards the equalization of the rate of profit accross sectors. Although it is not generally specified, this would appear to assume that the objective of firm is profit maximization in some sense. (SAWYER, 1994.a, p.215).

Para um maior esclarecimento do que será tratado a partir destas definições, é necessário investigar maiores considerações sobre o que envolve a construção teórica pós-keynesiana e clássica no que diz respeito à competição e à noção de longo prazo. 


\subsection{A concorrência clássica e a concorrência pós-keynesiana}

Segundo Sawyer (1994.b), a competição dentro da abordagem clássica envolve, para os propósitos de análise deste artigo, as seguintes características:

(1) a competição envolve rivalidade entre as firmas, esta rivalidade é conduzida por uma série de fatores relacionados à competição prices e non-prices. A intensidade da rivalidade entre as firmas não depende do número de firmas envolvidas, e a rivalidade pode ser particularmente feroz quando somente duas ou três firmas estão envolvidas;

(2) a mobilidade do capital na procura de lucros gera uma tendência em direção da equalização da taxa de lucro entre os setores. Esta tendência pode requerer um tempo de duração para completar-se.

Seguindo ainda Sawyer (1994.b), a competição dentro da abordagem pós-keynesiana envolve duas abordagens distintas: a primeira pode ser classificada como Kaleckiana (ou capitalismo de monopólio), e a segunda abordagem pode ser classificada como Eicheneriana (ou de investimento baseado na formação de preços).

Para os propósitos deste trabalho, estar-se-á apenas interessado na primeira abordagem, ou seja, a abordagem Kaleckiana que pode ser definida nos seguintes termos:

The theories of monopoly capitalism have their origins in the work of Hilferding (1910,1981) and Lenin (1916,1936), with Baran and Sweezy (1966) and Cowling (1982) being perhaps the most widely known contributions. The work of Kalecki and Steindl are also relevant here, and indeed we often to this approach as Kaleckian. Theories of monopoly capitalism have clear stagnationist implications, most evident in the work of Steindl $(1952,1976)$ and Cowling (1982, 1992), with economies exhibiting excess capacity operation and slow growth. Excess capacity, can be a result of inadequate aggregate demand but also has to be accepted by the enterprises since they can, through their investment decisions, vary the stock of capital equipament and could try to bring their stock of equipament into line with demand. They may accept some degree of excess capacity to accomodate fluctuations in demand and to use as a barrier to discourage new entrants. (SAWYER, 1994.b, p.10).

Uma vez estabelecidas as características da concorrência dentro das abordagens clássicas e pós-keynesianas, partiremos para as características de longo prazo para ambas as correntes em análise. 


\subsection{O longo prazo clássico na tradição neo-Ricardiana.}

A compreensão da proposta teórica neo-ricardiana pode ser preliminarmente definida nos seguintes termos:

Este grupo oferece uma interpretação da Teoria Geral de Keynes com base na análise de longo prazo clássica e vem estudando a adequação do sistema de preços de produção de Sraffa à teoria do emprego desenvolvida por Keynes. A motivação para este programa de pesquisa é dupla: em primeiro lugar proporcionar ao princípio da demanda efetiva de Keynes uma teoria do valor consistente; e, em segundo, desenvolver uma crítica à teoria marginalista da produção e do emprego, baseada que é no ajustamento entre poupança e investimento através de mudanças na taxa de juros. (AMADEO \& DUTT, 1987, p.562).

É amplamente conhecida a idéia de que Keynes, quando do desenvolvimento da Teoria Geral, negligenciou as questōes relativas ao longo prazo e, da mesma forma, rompeu de forma definitiva com a abordagem clássica. Tal procedimento, segundo a escola neo-ricardiana, implica uma deficiência na análise de Keynes quando da sua pretensão em fornecer uma teoria geral ${ }^{2}$. Logo, o objetivo da escola neo-ricardiana consiste em oferecer uma teoria baseada no princípio da demanda efetiva de Keynes, considerando as posiçōes de longo prazo, utilizando-se para tal, a recuperação de argumentos clássicos até então desconsiderados. Tais preocupações e objetivos podem ser esclarecidos nos termos abaixo:

... os neo-ricardianos procuram combinar, no contexto da análise de longo período, as preocupações clássicas com o valor e a distribuição, de um lado, com o princípio da demanda efetiva formulado por Keynes - igualdade entre poupança e investimento aquém do pleno emprego gerada por variações na renda, e não nos preços relativos - , de outro lado. Na sua avaliação, para que a teoria do produto e do emprego de Keynes efetivamente mereça o rótulo de geral, podendo, conseqüentemente, ser encarada como uma fecunda e consistente alternativa à teoria neoclássica do produto e do emprego, ela deve, necessariamente, referir-se às posições de longo período (centros de gravidade) do sistema de mercado. (LIMA, 1992, p. 84).

${ }^{2}$ Ver Eatwell $\mathcal{E}$ Milgate (1983.a). 
Desta forma, a abordagem neo-ricardiana procura estabelecer uma separação "entre as teorias da distribuição e dos preços relativos, de um lado, e da produção, emprego e acumulação, de outro" (AMADEO $\mathcal{E}$ DUTT, 1987 , p. 563). A tentativa de compatibilização da teoria clássica do valor e da distribuição e o princípio da demanda efetiva de Keynes como determinante do produto e emprego passam pela compreensão do trabalho de Piero Sraffa: Production of commodities by means of commodities. Sraffa propōe, seguindo a teoria clássica, que o processo de formaçã̃o dos preços é determinado pelas condiçōes de reprodução das mercadorias. Logo, a distribuição do excedente sobre os salários se dá a uma taxa de lucro "normal" sobre o valor do capital investido em cada indústria.

Quando consideradas as posições de longo prazo para a distribuição do excedente sobre os salários, verifica-se que tal condição requer a uniformidade da taxa geral de lucros para as indústrias. Esta uniformidade da taxa geral de lucro é obtida através das forças conduzidas pelo processo de competição entre as indústrias na busca do melhor retorno para os seus investimentos de capital. Quando obtida uma taxa de lucro uniforme para todos os possíveis projetos de investimento de capital, ou seja, em todas as indústrias, a economia encontrar-se-á em uma posição de longo prazo.

Conforme a escola neo-ricardiana, as teorias clássicas do valor e da distribuição "deixam abertas as questōes pertinentes à determinação do nível 'normal' de produto e emprego" (EATWELL $\mathcal{3}$ MILGATE, 1983, p. 7), mesmo porque tais questões devem ser determinadas separadamente. Logo, é perfeitamente possível vincular a teoria clássica da determinação do valor e da distribuição com a teoria keynesiana de determinação do emprego e do produto através do princípio da demanda efetiva. Uma deficiência da abordagem keynesiana, segundo os neo-ricardianos, consiste "em propor uma relação funcional entre a distribuição (a taxa de juros) e o montante de investimento na forma de eficiência marginal do capital [...] Keynes diminuiu a força do próprio argumento quando considera elementos da teoria neoclássica de valor e distribuição em suas discussões sobre os determinantes do volume de investimento." (EATWELL $\&$ MILGATE, 1983.a, p. 7).

Como já verificado, a abordagem neo-ricardiana consiste na análise do sistema econômico sob condiçōes de longo prazo. $O$ longo prazo neo-ricardiano consiste em uma situação de repouso na trajetória das variáveis relevantes do sistema ou "posições em direção às quais 
ou em torno das quais as variáveis relevantes do sistema gravitam." (AMADEO \& DUTT, 1987, p. 563).

Uma definição mais elucidativa do longo prazo neo-ricardiano pode ser tomada he empréstimo das noções de curto e longo prazos marshallianos, onde "quanto maior o período, maior a flexibilidade do sistema e, por conseguinte, em princípio, maior o número de variáveis endógenas." (AMADEO E 3 LiTT, 1987, p. 564).

No curto prazo marshalliano, observa-se a inflexibilidade do sistema econômico em que, dado o nível de estoques, o equilibrio de curto prazo consiste na configuração final das variações relacionadas aos fluxos da economia. Já no longo prazo marshalliano, o sistema econômico se flexibiliza de tal forma a permitir a variação dos estoques, ajustando-se desta forma ao equilibrio de longo prazo. Portanto, o equilíbrio de curto prazo marshalliano consiste no equilibrio de fluxos e o equilíbrio de longo prazo no equilibrio de estoques.

Para maiores esclarecimentos, vejamos mais algumas características do sistema marshalliano, nos seguinte: termos:

No curto prazo marshalliano, as posiçōes de desequilíbrio estāo associadas ao ajustamento da utilização da capacidade e do emprego. A posiçāo de equilíbrio corresponde a uma configuração final de emprego e utilização. Uma vez que a capacidade começa a ajustar-se à demanda (o longo prazo marshalliano), a análise passa a focalizar o processo de acordo com o qual o estoque de capital se modifica (através de uma série de posições de desequilíbrio) e a determinação da configuração final que inclui o tamanho e a estrutura setorial do estoque de capital. Em equilibrio, supõe-se que as empresas operem segundo o grau de utilização normal ou esperado. (AMADEO \& DUTT, 1987, p. 564 -565).

Seguindo a noção de longo prazo marshalliana, os neo-ricardianos consideram uma tendência da economia capitalista à plena utilização da capacidade produtiva ou a uma taxa "normal" de capacidade utilizada no longo prazo, porém cabe esclarecer tal significado, como segue definido abaixo:

By productive capacity we have so far meant the equipament of capital goods in existence in the economy in a given situation, together with only part of the total supply of labour which is required for the full utilisation of this equipament. By this definition we have departed from the terms in wich the controversy between Keynes and tradition theory was conducted. It was there assumed that a full utilisation of the existing capital equipament would allow for the employment on the entire labour force. (GAREGNANI, 1983, p. 24). 
Deste modo, a plena capacidade utilizada no longo prazo prescrita pelos neo-ricardianos implica duas proposições importantes: a) a plena utilização do equipamento de capital não garante o pleno emprego da força de trabalho; e b) a plena utilização do equipamento de capital é requerida para a uniformidade da taxa de lucro.

Vejamos mais detalhadamente esta segunda proposição, nos termos que seguem:

Uniformity of the rate of profit (between those firms employing the soccially necessary technique) requires that the scale and composition of output and the size and composition of capacity are adjusted one to the other. Thus the longrun normal position of the economy must embody a conception of the relation between output and capacity as a corollary of the conception of the normal relation between prices and distribution. (EATWELL, 1983.b, p. 271).

A proposição acima estabelece que uma posição "normal" de longo prazo é caracterizada por uma situação de uniformidade da taxa de lucro entre os vários setores da economia. Uma vez obtida a taxa de lucro "normal" do sistema, a relação normal entre preços e distribuição é dada. A obtenção desta taxa de lucro "normal" requer que a competição entre os setores estabeleça uma mobilidade do movimento de capitais em direção dos setores mais lucrativos. $O$ estado estacionário deste movimento é caracterizado quando o tamanho e a composição da capacidade ajusta-se ao nível de demanda agregada ou produto.

\subsection{O longo prazo pós-keynesiano na tradição Kalecki/Steindl}

Como é bem conhecido, o trabalho desenvolvido por Kalecki (1988) prescreve uma relação positiva entre o crescimento econômico e a distribuição da renda em favor dos salários. Para a obtenção de tal resultado, Kalecki admite que o sistema capitalista opere sob o regime de oligopólio, ou seja, os agentes são formadores de preços e utilizamse para a fixação dos mesmos de um mark-up sobre os custos unitários de produção. Associando tais fatores à existência de excesso de capacidade, pode-se obter a relação positiva entre crescimento e distribuição.

Kalecki analisa os efeitos que um aumento de salário (resultante da barganha dos sindicatos) exerce sobre o nível de emprego, nos termos que seguem:

... um aumento nos salários derivado do maior poder dos sindicatos conduz - contrariamente da percepção dos economistas clássicos - para um 
acréscimo no emprego. Contrariamente, uma queda nos salários derivada da perda de poder de barganha conduziria a uma queda no emprego." (KALECKI, 1988, p. 360).

Quando Kalecki pressupõe que os trabalhadores não poupam, e os capitalistas poupam uma fração dos lucros - uma distribuição da renda em favor dos salários teria o efeito de (a) reduzir o grau de monopólio e conseqüentemente o nível de mark-up, aumentando a participação dos salários no produto; e (b) aumentar o consumo dos trábalhadores e reduzir a capacidade excedente da economia. Este segundo efeito pode nos conduzir a uma verificação mais clara da relação positiva entre crescimento econômico e distribuição no longo prazo, quando consideramos o argumento de Steindl (1983), em que o nível de capacidade utilizada afetaria as decisões de investimento dos capitalistas.

A capacidade excedente considerada no trabalho de Steindl é aquela atribuída a uma situação de equilíbrio a longo prazo. Portanto, trata-se de uma situação mantida deliberadamente pelos produtores.

As justificativas dos produtores para a manutenção desta capacidade excedente seriam, segundo Steindl, as seguintes:

O primeiro motivo se apresenta facilmente, pela existência de flutuaçōes na demanda. O produtor deseja ser o primeiro a participar da fase de prosperidade, não deixando as vendas para os novos concorrentes, que pressionarão o seu mercado quando o periodo favorável se encerrar. Existe entretanto, um motivo mais profundo e geral. Qualquer produtor que constrói uma nova planta sabe que, durante um período inicial (que não devemos imaginar seja curto demais), ele poderá conquistar apenas um mercado restrito, devido à fidelidade dos consumidores e a toda uma série de fatores bem conhecidos. Não obstante, ele dimensionará a sua capacidade de modo a deixar bastante campo para uma produção maior, pois espera ser capaz de expandir suas vendas mais tarde. Essa esperança é fundamentada na experiência comprovada de que o crescimento do mercado (ou de sua "clientela") é uma função do tempo. (STEINDL, 1983, p. 23).

O segundo motivo acima relacionado é pertinente à incapacidade dos produtores de ajustarem ou expandirem sua capacidade de forma gradual, devido à indivisibilidade da oferta.

Quando Steindl supōe a existência de um nível de capacidade ociosa mantida deliberadamente conforme as aspirações dos produtores, ou seja, um nivel de capacidade excedente planejada, fica claro que tal qualificação supõe a existência de um segundo tipo de capacidade excedente não desejada. 
A distinção entre o nível de capacidade excedente planejada e a capacidade excedente não desejada se dá, segundo Steindl, conforme as decisōes de investimento dos produtores.

Um nível de capacidade excedente superior ou inferior ao planejado pelos produtores implicaria alguma reação por parte dos mesmos no sentido de eliminar tal inconveniente. Para Steindl, a reação mais provável é através do investimento e vejamos qual o argumento utilizado pelo autor para justificar tal proposição:

Que motivos temos para crer que a capacidade excedente não desejada desestimule o investimento? Em primeiro lugar, essa parece ser uma ilação natural do conceito de "capacidade excedente planejada". Se os empresários considerarem desejável certo volume de capacidade excedente, isso naturalmente significa que eles não aprovarão uma capacidade excedente maior ou menor. Significa, em outras palavras, que eles não são indiferentes em relação ao grau de utilização realmente obtido. A sua reação, caso desejem um ajuste, só pode compreender duas atitudes: podem procurar influenciar o mercado, por exemplo mediante uma redução nos preços, ou podem retardar ou acelerar o ritmo de investimento. O primeiro tipo de reação, sabemos, é muitas vezes impraticável. O segundo é sempre possível (embora nāo necessariamente muito adequado, a curto prazo). Deveria ocorrer, pelo menos, nos casos em que o primeiro tipo de reação não existe, e talvez deva ser o mais comum. (STEINDL, 1983, p. 147).

Vejamos como o nível de capacidade excedente não desejada afeta o investimento e como pode ser determinado teoricamente o nível de capacidade excedente planejada pelos produtores. Com tal finalidade, observamos como Steindl define sua função investimento, como segue:

$$
I_{t+\theta}=\gamma \frac{I}{K t}+u_{(u t)}+D_{(g t)}+r_{(p t)}
$$

Onde :

O primeiro termo, $\gamma \mathrm{I} / \mathrm{Kt}$, é simplesmente certa relação da acumulação interna no tempo $\mathrm{t}^{3}$. Os termos $\mathrm{u}, \mathrm{D}$ e r indicam a influência da utilização, da taxa de endividamento e da taxa de lucro no tempo $t$, respectivamente. A soma dessas influências determinará o investimento I após o tempo q. (STEINDL, 1983, p. 148-149).

${ }^{3}$ Conhecido na literatura keynesiana com animal spirit. 
Para os nossos propósitos, consideraremos constantes todos os determinantes da função investimento acima relacionada, excetuando-se o determinante relacionado à utilização $u_{(u t)}$.

Para um determinado nível de utilização $u_{(u t)}$, a influência deste sobre o investimento é nulo, ou seja, este nível corresponde ao da capacidade excedente planejada, que denominaremos por $\mathrm{u}^{*}{ }_{(\mathrm{ut})}$. Caso o nível de utilização fosse superior ao nível de capacidade excedente planejada $\mathrm{u}^{*}{ }_{(\mathrm{ut})}$, observaríamos $\mathrm{um}$ aumento no nível de investimentos. Da mesma maneira, observariamos uma redução no nível de investimentos caso o nível de utilização fosse inferior ao da capacidade excedente planejada $u^{*}$ (ut) , isso pode ser resumido da seguinte forma:

a) Para um nível de utilização igual ao de capacidade excedente planejada, temos:

$$
\frac{d I_{t+\theta}}{d u_{(u t)}}=0 \Rightarrow u_{(u t)}=u_{(u t)}^{*}
$$

b) Para um nível de utilização superior ao da capacidade excedente planejada, temos:

$$
\frac{d I_{t+\theta}}{d u_{(u t)}}<0 \Rightarrow u_{(u t)}<u_{(u t)}^{*}
$$

c) Para um nível de utilização inferior ao da capacidade excedente planejada, temos:

$$
\frac{d I_{t+\theta}}{d u_{(u t)}}>0 \Rightarrow u_{(u t)}>u_{(u t)}^{*}{ }^{4}
$$

\footnotetext{
${ }^{4}$ Observe que a sensibilidade do investimento em relação ao nível da capacidade utilizada dependerá do diferencial entre o nível efetivo e o nível planejado. No caso da equação (3) por exemplo, enquanto $u_{(u t)}<u_{(u t)}^{*}$ a variação do investimento sempre será negativa (mesmo com variações positivas do nivel da capacidade). Por esta razão, a teoria de Steindl é denominada "estagnacionista" e muito próxima das proposiçōes de Harrod e Domar, ou seja, $u_{(u t)}=u_{(u t)}^{*}$ é o "fio da navalha" do modelo de Steindl (considerando que as demais variáveis explicativas do investimento permaneçam constantes).
} 
Quando Steindl admite que o nível de utilização exerce influência sobre as decisões de investimento e sobre o processo de acumulação de capital, fica claro que uma melhora na distribuição da renda em favor dos salários estimularia o consumo (conforme o argumento verificado em Kalecki, em que os trabalhadores gastam tudo o que ganham, portanto não poupam) e este, por sua vez, estimularia o nível de utilização e, finalmente, o nível de investimentos e a acumulação do capital.

Deve-se lembrar que neste caso os lucros não serão comprometidos, uma vez que, como o aumentos dos salários estimulariam o investimento (via grau de utilização), o resultado seria um acréscimo dos lucros, uma vez que, segundo Kalecki, estes são determinados pelas despesas autônomas de investimento e consumo dos próprios.

\section{Controvérsias quanto a possibilidade de uma síntese}

O que se deseja nesta seção é verificar quais as tentativas em direção de uma possível síntese entre as noções de teoria de formação de preços e a teoria dos preços de produção, considerando ainda os elementos da concorrência Kaleckiana e os elementos da concorrência Clássica, respectivamente.

Uma destas tentativas foi elaborada por Dutt (1987), e seus elementos podem ser esclarecidos de forma reduzida, nos seguintes termos:

The centerpiece of Dutt's attempt to reconcile classical competition and equality of profit rates with monopoly theory is the development of "a model synthesizing the Sraffian prices of production framework and the Kaleckian-Steindl monopoly power theory. The Sraffian prices of production model is the static long-run representation of the classical prices of production equilibrium with equalized rates of profit. Dutt adds ti this framework the Kaleckian-Steindl theroy that excess capacity can exist in equilibrium anf that firm set prices by a markup over cost. Thus, Dutt's model combines markup prices and excess capacity mobility in a static equilibrium model. (CLICK \& CAMPBELL, 1995, p. 125).

Verificar-se-ão ainda nesta seção o modelo de Dutt e algumas críticas e demais argumentos contrários ao estabelecimento de qualquer tentativa de síntese desta natureza. 


\subsection{O Modelo de Dutt}

O modelo de Dutt considera uma economia fechada sem governo onde há dois setores, o setor 1 produzindo bens de consumo, e o setor 2 produzindo bens de capital. Cada bem requer capital e trabalho homogêneos, sem depreciação do capital. As relações de insumo-produto refletem coeficientes fixos e retornos constantes de escala e não há mudança técnica. Há duas classes: capitalistas e trabalhadores, os capitalistas auferem lucros sobre o capital e poupam uma fração constante destes lucros, os trabalhadores auferem salários monetários que são completamente despendidos na forma de consumo. Em termos algébricos, tais suposições podem ser estabelecidas da seguinte maneira:

$$
X_{1}=\left(\frac{W}{P_{1}}\right)\left(a_{01} X_{1}+a_{02} X_{2}\right)+(1-s)\left(\frac{P_{2}}{P_{1}}\right)\left(r_{1} K_{1}+r_{2} K_{2}\right)
$$

Na equação acima, o nível de produto $\left(\mathrm{X}_{\mathrm{1}}\right)$ do setor produtor de bens de consumo é definido pelo dispêndio dos trabalhadores e dos capitalistas. O primeiro termo da equação representa o dispêndio dos trabalhadores, onde $\mathrm{W} / \mathrm{P}_{1}$ é o nível de salário real dos trabalhadores em termos do nível de preços dos bens de consumo $\left(\mathrm{P}_{1}\right)$, multiplicado pela relação trabalho/produto $\left(\mathrm{a}_{01}\right.$ e $\left.\mathrm{a}_{02}\right)$ empregada em ambos os setores, respectivamente $\left(X_{1}\right.$ e $\left.X_{2}\right)$. $O$ segundo termo da equação representa o dispêndio dos capitalistas, onde (1-s) é a propensão a consumir dos capitalistas, multiplicada pela relação de termos de troca entre os dois setores $\left(\mathrm{P}_{2} / \mathrm{P}_{1}\right)$, multiplicado pelo retorno do capital para ambos os setores $\left(\mathrm{r}_{1} \mathrm{~K}_{1}+\mathrm{r}_{2} \mathrm{~K}_{2}\right)$, onde $\mathrm{r}_{\mathrm{i}}$ é a taxa de lucro e $\mathrm{K}_{\mathrm{i}}$ é o estoque de capital (i=1 e 2).

O nível de produto para o setor produtor de bens de capital $\left(\mathrm{X}_{2}\right)$ é definido conforme a equação abaixo descrita:

$$
X_{2}=g_{1} K_{1}+g_{2} K_{2}
$$

Na equação acima, $X_{2}$ é determinado pelas taxas de crescimento de estoques de capital de ambos os setores $\left(g_{1}\right.$ e $\left.g_{2}\right)$ multiplicado pelos seus respectivos níveis de estoques de capital $\left(\mathrm{K}_{1}\right.$ e $\left.\mathrm{K}_{2}\right)$.

Os preços são estabelecidos de forma a cobrir os custos de capital e trabalho:

$$
P_{1}=P_{2}\left(\frac{K_{1}}{X_{1}}\right) r_{1}+W a_{01}
$$


$\mathrm{Na}$ expressão acima, o primeiro termo da equação representa o custo do capital, ou seja, o preço do bem de capital $\left(\mathrm{P}_{2}\right)$ vezes a relação capital-produto utilizada na produção de bens de consumo $\left(\mathrm{K}_{1} / \mathrm{X}_{1}\right)$, multiplicado pela taxa de lucro do setor produtor de bens de consumo $\left(r_{1}\right)$. O segundo termo da equação representa o custo do trabalho, onde temos os salário nominal dos trabalhadores (W), multiplicado pela relação trabalho-produto utilizada na produção de bens de consumo $\left(\mathrm{a}_{01}\right)$.

Da mesma forma é determinado o nível de preços do setor produtor de bens de capital, ou seja:

$$
P_{2}=P_{2}\left(\frac{K_{2}}{X_{2}}\right) r_{2}+W a_{02}
$$

O primeiro termo da equação representa o custo do capital, ou seja, o preço do bem de capital $\left(\mathrm{P}_{2}\right)$, vezes a relação capital-produto utilizada na produção de bens de capita $\left(\mathrm{K}_{2} / \mathrm{X}_{2}\right)$, multiplicado pela taxa de lucro do setor produtor de bens de capital $\left(\mathrm{r}_{2}\right)$. O segundo termo da equação representa o custo do trabalho, onde temos o salário nominal dos trabalhadores (W) vezes a relação trabalho-produto utilizada na produção de bens de capital $\left(\mathrm{a}_{02}\right)$.

Considera-se ainda que os preços sejam estabelecidos conforme a noção pós"keynesiana de formação de preços, ou seja, as firmas fixam seus preços de acordo com um markup sobre seus custos unitários:

$$
P_{i}=\left(1+z_{i}\right) W a_{0 i}
$$

Onde $z_{i}(i=1$ e 2 ) é a taxa de markup no setor $\mathrm{i}$, dado para cada setor conforme o grau de monopólio, que depende de fatores como a concentração industrial, a natureza da relação entre as firmas, o poder de barganha das empresas e sindicatos trabalhistas e o grau de uso do capital fixo.

A decisão de investimento das firmas depende da taxa de lucro e do nível da capacidade utilizada tal função é assumida por uma forma linear e pode ser expressa em termos algébricos da seguinte maneira:

$$
g=\frac{\left(g_{1} K_{1}+g_{2} K_{2}\right)}{\left(K_{1}+K_{2}\right)}=\tau_{0}+\beta_{1} u+\beta_{2} r
$$


Onde $\tau_{0}$ (animal spirits) e $\beta_{\mathrm{i}}$ são parâmetros fixos e positivos e onde :

$$
\begin{aligned}
& u=\left(\frac{P_{1}}{P_{2}}\right)^{0} u_{1} k_{1}+u_{2} k_{2} \\
& r=r_{1} k_{1}+r_{2} k_{2} \\
& \mathrm{u}_{\mathrm{i}}=\mathrm{X} / \mathrm{K}_{\mathrm{i}}, \mathrm{k}_{\mathrm{i}}=\mathrm{K} /\left(\mathrm{K}_{1}+\mathrm{K}_{2}\right), \mathrm{e}_{\mathrm{i}}>0 \text { são parâmetros fixos e onde (P1) }
\end{aligned}
$$
$\mathrm{P} 2)^{0}$ denota a razão de preços inicial.

Assumindo que a alocação do investimento entre os dois setores é conduzinda pelo diferencial das taxas de lucros, ou seja, partindo da proposição neo-ricardiana que a equalização da taxa de lucro no longo prazo entre os setores é estabelecida mediante o fluxo de capital de curto prazo para setores com maior atratividade, temos que:

$$
g_{1}-g_{2}=\mu\left(r_{1}-r_{2}\right)
$$

Onde $\mu>0$. No curto prazo é assumido que, dado o nível de estoque de capital, o mercado para cada bem seja ajustado mediante variações no nível do produto em resposta aos excessos de demanda.

Do conjunto de equações acima descritas, podemos obter os valores de equilíbrio de curto prazo para os níveis de capacidade utilizada $\left(\mathrm{u}^{*}{ }_{1} \mathrm{e} \mathrm{u}_{2}^{*}\right)$, das taxas de lucro $\left(\mathrm{r}^{*}{ }_{1}{\mathrm{e} \mathrm{r}_{2}^{*}}_{2}\right)$, do nível de preços relativos $\left(\mathrm{P}^{*}{ }_{2} / \mathrm{P}^{*}{ }_{1}\right)$, do salário real $\left(\mathrm{W}^{*} / \mathrm{P}^{*}{ }_{1}\right)$ e do nível de acumulação do estoque de capital $\left(\mathrm{g}^{*}\right)^{5}$ :

$$
\begin{aligned}
& u_{1}^{*}=\left(\frac{a_{02}}{a_{01}}\right)\left[\left\{\frac{1+(1-s) z_{2}}{\left(1+z_{1}\right)}\right]\right\} \tau_{0} \frac{(1+k)}{k \Sigma} \\
& u_{2} *=\frac{(1+k) s\left[\frac{z_{1}}{\left(1+z_{1}\right)}\right] \tau_{0}}{\Sigma}
\end{aligned}
$$

${ }^{5}$ Os asteriscos representam os valores de equilibrio das variáveis. Consultar apêndice matemático para maiores esclarecimentos quanto ao processo de obtenção de tais valores. 


$$
\begin{aligned}
& \left.r_{1}^{*}=\left[\frac{z_{1}}{\left(1+z_{2}\right)}\right] \frac{1+(1-\mathrm{s}) \mathrm{z}_{2}}{\left(1+z_{1}\right)}\right] \tau_{0} \frac{(1+\mathrm{k})}{\mathrm{k} \Sigma} \\
& r_{2}^{*}=\left[z_{2}\left(1+z_{2}\right)\right] \frac{(1+k) s\left[\frac{z_{1}}{\left(1+z_{1}\right)}\right] \tau_{0}}{\Sigma} \\
& \frac{P_{2}^{*}}{P_{1}}=\left[\frac{\left.\left(1+z_{2}\right)\right]\left(\frac{a_{02}}{a_{01}}\right)}{\left.\left(1+z_{1}\right)\right]}\right. \\
& \frac{W^{*}}{P_{1}}=\frac{1}{\left(1+z_{1}\right) a_{01}} \\
& \mathrm{~g}^{*}=\tau_{0}\left\{\frac{1+\tau_{1}\left(\frac{\mathrm{a}_{02}}{\mathrm{a}_{01}}\right)[1+(1-\mathrm{s})] \mathrm{z}_{2}+\tau_{2} \mathrm{sz}_{1}}{\mathrm{sz}\left(1-\tau_{2}\right)-\tau_{1}\left(\frac{\mathrm{a}_{02}}{\mathrm{a}_{01}}\right)\left[1+(1-s) \mathrm{z}_{2}\right.}\right]
\end{aligned}
$$

Deve-se observar que todos os valores de equilibrio de curto prazo para as variáveis acima relacionadas são estabelecidos em função dos markups $\left(z_{1}\right.$ e $\left.z_{2}\right)$, dos coeficientes tecnológicos $\left(a_{01}\right.$ e $\left.a_{02}\right)$ e das constantes do modelo, ou seja, neste modelo todas as variáveis relevantes são determinadas pelos graus de monopólio setoriais que, por sua vez, são determinados pela natureza de rivalidade entre as firmas e o equilibrio de poder entre diferentes firmas e trabalhadores. Nestes termos, tal modelo desprende-se das proposições clássicas de concorrência e caminha em direção das proposições pós-keynesianas de concorrência oligopolística e formação de preços.

No entanto, a característica Clássica do modelo de Dutt repousa sobre as condições de equilibrio de longo prazo, ou em termos neoricardianos, de longo período.

No longo prazo, o estoque de capital nos dois setores muda de acordo com as equações (10) e (11). A dinâmica de longo prazo do 
modelo pode ser examinada com as seguintes equações de reação, como segue:

$$
\dot{k}=g_{1}-g_{2}
$$

Onde o ponto sobre a variável denota sua taxa de crescimento no tempo. A partir da equação (11), sabe-se que

$$
\dot{k}=\mu\left(r_{1}-r_{2}\right)
$$

Onde a equação (20) juntamente com as equações (12) e (15) definem a dinâmica do sistema envolvendo $\mathrm{k}$. $\mathrm{O}$ equilíbrio de longo prazo de $\mathrm{k}$ é definido por $\mathrm{k}^{*}=0$, onde o valor de $\mathrm{k}$ é estacionário.

As equações (12) e (14) implicam que $\mathrm{dr}_{1} / \mathrm{dk}<0$, enquanto as equações (13) e (15) implicam que $\mathrm{dr}_{2} / \mathrm{dk}_{2}>0$; isto implica que $\mathrm{dk}^{*} / \mathrm{dk}$ $<0$, ou seja, a solução de equilibrio é estável, ou ainda, o sistema observa apenas uma única solução estável, conforme pode ser verificado no gráfico a seguir.

\section{Gráfico: Dinâmica da equalização da taxa de lucro}

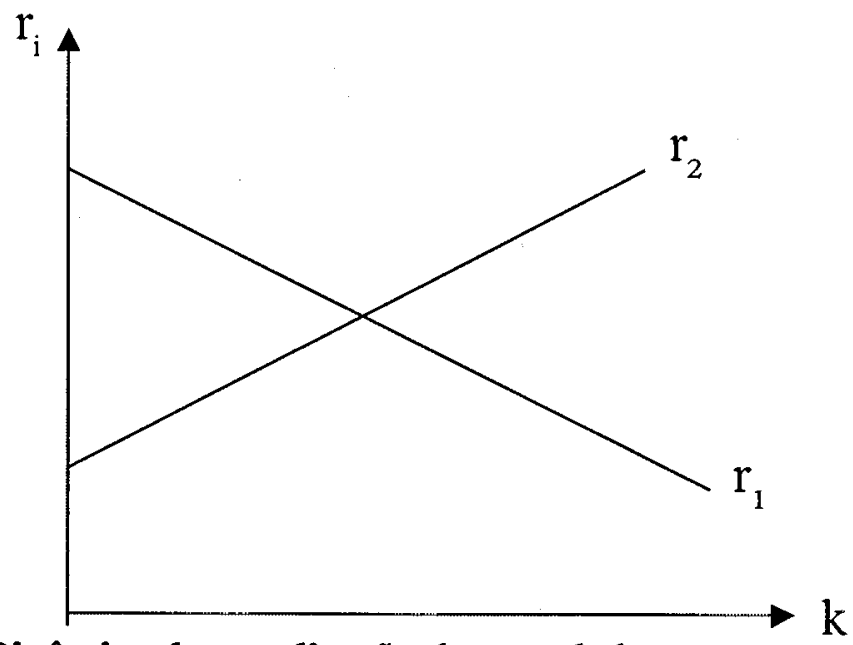

A dinâmica do sistema pode ser observada ainda nos seguintes termos:

Starting from any initial value of $k$, the economy will move to a long-run equilibrium at which, as can be seen from equation (20), $r_{1}=r_{2}$. Thus, given any starting point, the economy will converge to a balanced growth path with profit rates equalized between sectors. Once the long-run equilibrium value of $k$ is determined, this value 
can be substituted into equations (12) and (13) to obtain the long-equilibrium values of $u_{i}$. The equilibrium value of the rate of profit can then be found from equation (14) or (15). The long -run equilibrium values for the relative price, real wage, and the growth rate are given by level already found in equation (16), (17) and (18); they do not change during the adjustment to long-run equilibrium. (DUTT, 1995, p. 145).

O ponto de equilibrio é obtido da seguinte maneira: Como $\mathrm{dr}_{1} / \mathrm{dk}$ $<0$, temos uma relaçāo inversa estabelecida entre $\mathrm{r}$ e k; e como dr $\mathrm{d}_{\mathrm{f}}$ $\mathrm{dk}>0$, temos uma relação positiva entre as mesmas variáveis; logo, a intersecção das curvas $r_{1}$ e $r_{2}$ em um espaço $\left(r_{i} \times k\right)$ estabeleceria a situação de equilíbrio.

\subsection{Críticas ao modelo de Dutt}

A partir de agora, estar-se-á interessado em verificar as críticas quanto à possibilidade de uma eventual síntese, nos moldes estabelecidos por Dutt. Inicialmente verificar-se-ão algumas críticas quanto a consistência e o realismo do modelo de Dutt.

Um primeiro conjunto de críticas é estabelecido por um grupo de economistas neo-ricardianos, cujo o conteúdo pode ser resumidamente verificado nos seguintes termos:

In our view Dutt has failed to develop na economically realistic model in which monopoly capital is compatible with equalized rates of profit. In Dutt's model, the unrealistic assumption that drives the results is that, regardless of the markup chosen by each firm, capacity utilization adjusts (in just the right way) to achieve an equalized rate of profit. We have pointed out a number of problems with this mechanism. First, Dutt's model itself cannot produce the differential investment that would be necessary for the adjustment process he verbally suggest. Second, a short-run capacity adjustment mechanism requires na ad hoc assumption concerning the structure of demand. Finally, the suggested adjustment mechanism of capital moving into high profit sectors without affecting the ability of the incumbent firm to maintain the markup and hence price (which means the amount of monopoly power is not affected by this inflow of capital) is inconsistent with real world competitive rivarly. (CLICK 8 CAMPBELL, 1995, p.133).

Considerando ainda as críticas ao modelo de Dutt, um argumento é desenvolvido por Park (1995), em que argumenta:

... there is na internal inconsistency in Kaleckian models with several sectors, [...] in which markup pricing holds in each sector and investmentin each of two 
sectors is assumed to be a function of that sector's rate of profit and capacity utilization rate. When one introduce na equation which requires profit rate equalization accross sectors, the model becomes over-determined. (apud DUTT, 1999, p. 42).

Um segundo conjunto de considerações envolve a questão da impossibilidade de uma completa sintetização destas abordagens e não constituem uma crítica direta ao modelo de Dutt. Estas questões são relevantes pois estabelecem as diferenças de ambas as abordagens quanto à participação dos lucros no produto, logo, tais considerações envolvem questões relativas à distribuição da renda, que serão objeto de análise da próxima seção. Tais considerações podem ser expressas nos seguintes termos:

The classical view is that the profit share by reference to the subsistence wage and that the capacity utilization (in the long period) will be unity. The Kaleckian view would be that the share be determined by the (average) 'degree of monopoly' where the latter is broadly interpreted to include power of workers to influence the markup. Capacity utilization can be variously seen as influenced by the level of aggregate demand and by the objectives and the interests of the firms. There are clear remaining differences here over whether the long term can involve excess capacity, and hence whether some of the adjustment towards uniformity of the rate of profit can come through variations in capacity utilization. At the level of the firm there are many routes through which high profits can be dissipated: for example, managerail salaries, productive inneficiency. (SAWYER, 1994, p. 18-19).

Com as considerações acima, pode-se estabelecer um ponto fundamental quanto a controvérsia estabelecida entre a possibilidade de uma síntese entre a teoria dos preços de produção e a teoria de formação de preços kaleckiana, a saber, o nível de utilização da capacidade no longo prazo (que diverge em cada uma destas abordagens). Por trás desta divergência é que repousa a maior controvérsia entre a abordagem pós-keynesiana na tradição Kalecki/Steindl e a abordagem Clássica na tradição marxista ou neo-ricardiana: a relação entre o crescimento econômico e a distribuição funcional da renda no longo prazo.

\subsection{O ponto nevrálgico da controvérsia}

A questão central da controvérsia que envolve a possibilidade de uma síntese da teoria de formação de preços kaleckiana, baseada na concorrência imperfeita e formação de preços por markup, e uma teo- 
ria dos preços sraffiana com mobilidade do capital e equalização das taxas de lucros repousa sobre a configuração do nível da capacidade utilizada no longo prazo.

Como já verificado anteriormente, a teoria kaleckiana advoga a possibilidade de um nível de capacidade ociosa de longo prazo que, conforme Steindl, consistiria de uma capacidade planejada com os objetivos de dissuadir a concorrência e devido às indivisibilidades da planta.

Quanto aos neo-ricardianos (ou sraffianos), estes advogam a inevitabilidade da plena utilização da capacidade no longo prazo (embora isto não envolva a plena utilização da força de trabalho). Tal proposição é realmente inevitável dentro deste arcabouço teórico, uma vez que, o longo prazo neo-ricardiano consiste na plena flexibilidade das variáveis de estoques. Deste modo, o estoque de capital no longo prazo é flexível o suficiente para ajustar-se ao nível da demanda agregada, configurando, assim, a plena utilização do estoque de capital. Tal questão pode esclarecer o argumento de Eatwell onde este estabelece que "a uniformidade da taxa de lucro (entre as firmas que empregam a técnica socialmente necessária) requer que a escala e a composição do produto e o tamanho e a composição da capacidade sejam ajustadas uma à outra."

Eatwell continua o argumento acima considerando que "uma posição normal de longo prazo para a economia deve conceber a concepção da relação entre produto e capacidade como um corolário da concepção da relação normal entre preços e distribuição". Logo, podese observar claramente que a configuração da capacidade utilizada do equipamento de capital no longo prazo implica uma questão de suma importância, a saber, a relação entre o crescimento econômico e a distribuição da renda no longo prazo, questão esta que, conforme Ricardo (1996), constitui-se na "principal questão da Economia Política".

Vejamos, então, como a utilização da capacidade utilizada no longo prazo estabelece a relação entre o crescimento econômico e distribuição funcional da renda em cada uma destas teorias.

(a) Capacidade utilizada, crescimento econômico e distribuição da renda no longo prazo: a abordagem pós-keynesiana na tradição Kalecki/Steindl.

Conforme já verificado nas seções anteriores, os modelos póskeynesianos na tradição Kalecki/Steindl estabelecem que a economia opera com capacidade ociosa no longo prazo. Verificou-se ainda que os desvios do nível da capacidade efetiva em torno da capacidade plane- 
jada influiriam nas decisões de investimento e acumulação das firmas. Deste modo, a função acumulação poderia ser expressa para cada firma individual da seguinte maneira:

$$
\frac{I}{K}=\beta_{0}+\beta_{1} r+\beta_{2} u
$$

Onde $\beta_{\mathrm{i}}, \mathrm{i}=1,2,3$ são parâmetros positivos, $\mathrm{r}$ é a taxa de lucro e $\mathrm{u}=\mathrm{X} / \mathrm{K}$ é o nível da capacidade utilizada. Seguindo a tradição kaleckiana de que os trabalhadores gastam toda a sua renda em consumo e os capitalistas poupam uma fração constante de seus lucros, fica evidente que uma distribuição da renda em favor dos salários geraria um aumento da demanda agregada, que por sua vez estimularia o nível da capacidade utilizada e o investimento e os lucros capitalistas, uma vez que, os capitalistas ganham o que gastam.

(b) Capacidade utilizada, crescimento econômico e distribuição da renda no longo prazo: a abordagem neo-ricardiana na tradição Clássica.

Quanto aos neo-ricardianos, a plena utilização da capacidade no longo prazo envolve um trade-off entre o crescimento econômico e a distribuição da renda em favor dos salários, seguindo assim os pressupostos de economistas Clássicos como Marx e Ricardo.

Uma crítica neo-ricardiana aos kaleckianos envolve a possibilidade de haver capacidade ociosa no longo prazo, assunto este já destacado anteriormente. Um segundo conjunto de críticas envolve a questão da capacidade utilizada poder influenciar as decisões de investimentos da firma. Esta crítica pode ser resumidamente expressa da seguinte maneira:

The view of both Ciccone (1986, p.26) and Vianello (1989), and also of Garegnani (1992, p.56) and Kurz (1992, p.22), is that new investment depends on expected profitability, computed at normal prices based on the normal rate of utilisation of capacity. This means that the investment function depends on the (expected) normal rate of profit rather than on the actual rate of profit. The justification for this is that entrepreneurs cannot make future plans under the assumption that capacity will be perpetually over-utilised. Plans must be made according to profitability at normal use of capacity. The rate of profit that represents 'the guiding light for investment and pricing decisions, cannot possibly be either an abnormally high or an abnornally low one' (Vianello, 1985, p.85). (LAVOIE, 1995, p. 796).

Desta forma, a função acumulação da firma, segundo a escola neo-ricardiana, poderia ser expressa da seguinte maneira: 


$$
\frac{I}{K}=\beta_{0}+\beta_{1} r^{e}
$$

Onde $\beta_{\mathrm{i}}, \mathrm{i}=1,2$ são parâmetros positivos, $\mathrm{r}^{\mathrm{e}}$ é a taxa esperada de lucro. Como a "rentabilidade esperada é computada a preços normais e baseadas em uma taxa normal de capacidade utilizada", temos que $\mathrm{u}=\mathrm{X} / \mathrm{K}=1$, sendo $\mathrm{u}$ uma constante, logo nāo influencia as decisões de investimento da firma. Dado o nível normal da capacidade, um aumento da taxa de salários não teria efeito sobre o produto e diminuiria pro tanto a taxa de lucro, reduzindo, assim, o investimento e a taxa de acumulação.

\section{Conclusão}

As várias tentativas de sintetizar uma teoria de formação de preços kaleckiana com uma teoria dos preços de produção sraffiana enfrentam várias controvérsias e críticas.

A principal controvérsia repousa sobre a questão da configuração da utilização da capacidade no longo prazo, uma vez que esta configuração determinará a relação entre a acumulação de capital e a distribuição da renda.

Modelos como o desenvolvido por Dutt, que estabelece formação de preços por markup e capacidade ociosa de longo prazo, associado com a noção Clássica de longo prazo com equalização da taxa de lucros entre os setores encontram forte resistência, uma vez que deturpam uma característica intocável da Escola Clássica, a saber: a oposição frontal entre lucros e salários e conseqüentemente a oposição frontal entre distribuição e crescimento. 


\section{ANEXO}

Os valores de equilíbrio do modelo são obtidos através da transformação da equação (10), a partir da combinação desta com as equações (7) e (9), obtendo-se assim a seguinte equação abaixo:

$$
g=\tau_{0}+r_{1} k_{1} u_{1}+\tau_{2} k_{2} u_{2}
$$

Onde

$$
\begin{aligned}
& \tau_{1}=\beta_{1}\left(\frac{a_{01}}{a_{02}}\right)\left[\frac{\left(1+z_{1}\right)}{\left(1+z_{2}\right)}\right]+\beta_{2}\left(\frac{a_{02}}{a_{01}}\right)\left[\frac{\left(1+z_{2}\right)}{z_{1}}\right] \\
& \tau_{2}=\beta_{1}+\beta_{2} \frac{\left(1+z_{2}\right)}{z_{1}} \\
& k=\frac{K_{1}}{K_{2}}
\end{aligned}
$$

As equações (12) e (13) são obtidas através das equações (5), (9) e (10'), onde:

$$
\Sigma=s\left[\frac{z_{1}}{\left(1+z_{1}\right)}\right]\left(1-\tau_{2}\right)-\tau_{1}\left(\frac{a_{02}}{a_{01}}\right) \frac{\left[1+(1-s) z_{2}\right]}{\left(1+z_{1}\right)}
$$

As equaçōes (7), (9), (12) e (13) determinam os valores das equações (14) e (15).

A substituição das equações (12) e (13) em (10') define o valor da equação (18). 


\section{Referências Bibliográficas}

AMADEO, Edward. J. (1986). "Notes on Capacity Utilisation, Distribution and Accumulation". Contributions to Political Economy, 5, 83-94.

AMADEO, Edward. J.; DUTT, Amitava K. (1987). "Os Keynesianos neoRicardianos e os pós-Keynesianos”. Pesquisa e Planejamento Econômico, 17, 561604.

ASIMAKOPULOS, A. (1988). "A Kaleckian Theory of Income Distribution". In M. Sawyer (ed) Post-Keynesian Economics. Aldershot, Edward Elgar.

AUERBACH, P.; SKOTT, P. (1988). "Concentration, Competition and Distribution". International Review of Applied Economics, 2, 42-61.

CICCONE, R. (1986). "Accumulation and Capacity Utilization: Some Critical Considerations on Joan Robinson's Theory of Distribution". Political Economy, 2, 17-36.

DUTT, Amitava K. (1984). "Stagnation, Income Distribution and Monopoly Power". Cambridge Journal of Economics, 8, 25-40.

. (1987). "Alternative Closures Again : a Comment on 'Growth, Distribution and Inflation'”. Cambridge Journal of Economics, 11, 75-82.

. (1987a) "Competition, Monopoly Power and the Uniform Rate of Profit". Review of Radical Political Economics, 19, 55-72.

. (1990). Growth, Distribution, and Uneven Development. Cambridge, Cambridge University Press.

(1994). "Classical Competition and Post-Keynesian Monopoly Power: A Possible Synthesis". In M.A.Glick (ed), Competition, Technology and Money, Aldershot, Edward Elgar.

. (1999). "Kalecki and the kaleckians: the relevance of Kalecki today". In Anais: Seminário Internacional Dinâmica Econômica do Capitalismo Contemporâneo: uma Homenagem ao Centenário de Michal Kalecki, São Paulo, USP.

EATWELL, John; MILGATE, Murray. (1983.a). Keynes's Economics and the Theory of Value and Distribution. New York, Oxford University Press.

EATWELL, John, (1983b). "The Long-Period Theory of Employment". Cambridge Journal of Economics, 7, 269-285.

GAREGNANI, Pierangelo. (1980). "Sobre a Teoria da Distribuição e do Valor em Marx e nos Economistas Clássicos". In Unicamp (ed), Progresso Técnico e Teoria Econômica. São Paulo, Editora Hucitec.

(1983). "Notes on Consumption, Investment and Effective Demand". In

J. Eatwell 8 M. Milgate (eds), Keynes's Economics and the Theory of Value and Distribution. New York, Oxford University Press. 
. (1992). "Some Notes for an Analysis of Accumulation". In Halevi, J., Laibman, D. and Nell, E . (eds), Beyond the Steady State. A Revival of Growth Theory, London, Macmillan.

GLICK, Mark; CAMPBELL, D.A. (1995). "Classical Competition and the Compatibility of Market Power and Uniform Rates of Profit". Review of Radical Political Economics, 27, 124-153.

KALECKI, Michal. (1985). Teoria da Dinâmica Econômica. Os Economistas; São Paulo, Nova Cultural.

. (1988). "Class, Struggle and Distribution of National Income". In M. Sawyer (ed) Post-Keynesian Economics. Aldershot, Edward Elgar.

KURZ, H.D. (1986) “Normal Positions and Capital Utilization". Political Economy, 2, p.37-54.

. (1991). "Technical Change, Growth and Distribution: a Steady State Approach to Unsteady Growth on Kaldorian Lines". In Nell, E.J. and Semmler,W. (eds), Nicholas Kaldor and Mainstream Economics, London, Macmillan.

LAVOIE, Marc. (1995). "The Kaleckian Model of Growth and Distribution and its neo-Ricardian and neo-Marxian Critiques". Cambridge Journal of Economics, 19, 789-818.

LIMA, Gilberto T. (1992). Em Busca do Tempo Perdido: A Recuperaçāo pósKeynesiana da Economia do Emprego de Keynes. Rio de Janeiro, BNDES.

MARGLIN, Stephen A. (1984). "Growth, Distribution, and Inflation: a Centennial Synthesis". Cambridge Journal of Economics, 8, 115-144.

$\overline{\text { Press. }}$

. (1984.a). Growth, Distribution, and Prices. Cambridge, Harvard University

PARK, Man-Seop. (1995). A note on the 'Kaleckian-Steindl' steady state approach to growth and income distribution. The Manchester School, 63(3), 297-310.

RICARDO, David. (1996). Princípios de Economia, Política e Tributação. Os Economistas; São Paulo, Nova Cultural.

ROWTHORN, R. (1988) "Demand, Real Wages and Growth". In M. Sawyer (ed) Post-Keynesian Economics. Aldershot, Edward Elgar.

SAWYER, Malcolm. (1994a) "Prices, Capacity Utilization and Employment in the post-Keynesian Traditions". In A.K. Dutt (ed), New Directions in Analytical Political Economy, Aldershot, Edward Elgar.

(1994.b) "Post-Keynesian and Marxian Notions of Competition: Towards a Synthesis". In M. A. Glick (ed), Competition, Technology and Money, Aldershot, Edward Elgar.

SRAFFA, Piero. (1977) Produção de Mercadorias por Meio de Mercadorias. Rio de Janeiro, Zahar Editores. 